\title{
Distributed Time-Management in Transputer Networks
}

\author{
W.A. Vervoort, R. te West, A.L. Schoute, J. Hofstede \\ Department of Computer Science \\ University of Twente, The Netherlands.
}

\begin{abstract}
For real-time applications in a distributed system a common notion of time is indispensable. Clocks are used for time measurement, determination of causality, process synchronization and generating unique identifications. All this is only possible if there is a time reference of specified accuracy. Since the local clocks in a distributed system tend to drift away from each other, they need to be adjusted periodically. If the application allows an accuracy that can be met by software, this may be achieved by a distributed clock synchronization algorithm, which creates and maintains a global time reference for all nodes of the network. The design and simulation of such an algorithm for a distributed system consisting of transputers is the subject of this paper. It is based on second order filtered adjustment of the clock rates rather than updating the clock values at once.
\end{abstract}

Keywords:

transputer network, clock synchronization, second order filter, clock rate adjustment, simulation, distributed algorithms.

\section{Introduction}

Consider a distributed system in which each node has its own independent physical clock. Each node i maintains a logical clock $\mathrm{C}_{\mathrm{i}}(\mathrm{t})$ based on the physical clock, by adding a time dependent offset. As physical clocks are not accurate they tend to drift away from each other. We assume their maximum drift to be $\rho$ (typically for a transputer $\left.|\rho|<10^{-4} \mathrm{sec} / \mathrm{sec}\right)$. After $R$ seconds the maximum difference between two clocks could be $2 \rho R$ sec. This may be too large for a particular application. Depending on $\rho$ a reasonable resynchronization interval $\mathrm{R}$ has then to be defined after which the logical clocks are adjusted (by correcting their offset with the physical clock), in such a way that the clock value differences remain bounded by a known constant $\Delta$. This is the task of a clock synchronization algorithm.

In literature many clock synchronization algorithms are presented, designed for fully connected networks with predictable message delays. None of these are directly applicable to transputer networks for two reasons: arbitrary network diameters and unpredictable message delays.

\section{Network diameter.}

The diameter $\mathrm{d}$ of a network is the smallest number such that any two nodes in the network are connected by a path containing at most d links. A network which is fully connected has a diameter $\mathrm{d}=1$. Networks composed of transputers are in general not fully connected since a transputer has a limited number of links. The networks currently used are constructed with T414 or T800 transputers which have four links.

Message delay.

In order to keep the local clocks synchronized, it is necessary to determine at least some other nodes' clock times. In a distributed system a node can generally not read these directly. Instead messages have to be sent by clock keeping processes to exchange clock times. As communication is executed by a rendezvous mechanism a clock process may have to wait for a link to become ready. If another high priority process was running at the moment the link became ready the clock process is added to the end of the high priority process queue, so in general there is no upperbound on the message delay. As a result the time contents of the message is not accurate. This uncertainty accumulates if the message has to pass intermediate transputer nodes. 


\section{Design of an algorithm}

\section{Requirements}

Requirements for a clock synchronization algorithm as formulated in [Kopetz 87] state that the algorithm should maintain a maximum difference, bounded by a known constant, and a chronoscopic global time base. Moreover it should be fault tolerant and efficient.

Stated more precisely we want the algorithm to achieve two requirements:

(1) Keep the local (logical) clocks $C_{i}(t)$ such that for all $i$ and $j$ and at any real-time $t:\left|C_{i}(t)-C_{j}(t)\right| \leq \Delta$. As the accuracy should only depend on the diameter $d$ and not on the total number of nodes in the network, we will (rather arbitrary) chose $\Delta$ to be $0.1 \mathrm{~d} \mathrm{msec}$.

(2) In order to maximize resynchronization intervals, resulting in less communication overhead, we want the algorithm also to decrease the drift differences.

\section{Phases and functions}

In short, clock synchronization is based on comparing periodically some of the other clocks in the system with the own local clock and using this information to adjust the local clock such that a good approximation of some (imaginary) common time base (called: global time) is obtained.

Three phases of clock synchronization can be recognized [Thambidurai 89]:

- cold start (formation of a synchronized set of clocks starting with arbitrary initial clock states),

- warm start (integration of a new or repaired "fresh" node such that it becomes part of an existing set of synchronized nodes), and

- steady state (preservation of a set of synchronized clocks).

A clock synchronization algorithm is a distributed algorithm executing on each node of the network. It executes four functions in sequence:

- determination of the resynchronization moment,

- determination of (some) remote clock values,

- estimation of the global time, and

- adjustment of the local clock.

For each function a number of possible solutions exist which may depend on the three phases mentioned above.

\section{Definitions}

The following symbols are used: $i, j$ : nodes of the network

$t, t_{\text {send }}, t_{\text {rec }}, t_{\text {mid }}$, now : real-time moments

$d_{\text {min }}, d_{\text {send }}, d_{\text {rec }}, d_{\text {both }}:$ message delays

$C_{i}(t)$ : the clock value of node $i$ at $t$

$\rho_{i}(t)$ : drift of the clock of node $i$ at $t$

$C_{j i}(t)$ : i's estimation of $C_{j}(t)$ at $t$

$\varepsilon_{\mathrm{ji}}(\mathrm{t}): \mathrm{C}_{\mathrm{ji}}(\mathrm{t})-\mathrm{C}_{\mathrm{i}}(\mathrm{t})$

$\varepsilon_{i}(t)$ : (weighted) mean of $\varepsilon_{j i}(t)$ for $j \in$ neighbours of $i$

\section{Determination of resynchronization moment}

Possibilities: Two different approaches can be distinguished: central and distributed. In the central approach one node acts as a master for the whole network. It broadcasts the moment of resynchronization [De Carlini 88]. In the distributed approach the moment may be determined in two ways: all nodes may have agreed upon the same real-time moment and look for it at their own local clocks [Srikanth 87] or each node individually and independently of the others determines its next resynchronization moment [Lamport 85].

Choice: For all phases the second alternative of the distributed approach will be used because some nodes may want to use larger resynchronization intervals than others. In the cold and warm start a small interval is preferred in order to bring the nodes quickly together. In the steady state, as soon as the rates will have been brought closer together a larger interval may be possible.

\section{Determination of (some) remote clock values}

Possibilities: Two approaches are possible: global and local. In the global approach each node collects all other remote clock values, but in the local approach only some subset is collected (e.g. the direct neighbours).

Determination of a remote clock is done by periodically sampling it and using these samples to reconstruct its time. One or more of these samples per resynchronization interval are possible.

The message delay determines the accuracy. It can be estimated by measuring the time between a time-request message and the answer [Christian 89]. A confidence weight factor can then be associated with the received clock value, based upon the length of the total message delay. The algorithm of [Meijerink 89], based on more samples per interval, uses linear regression to eliminate the unknown message delay and to estimate the remote clock value as well as its drift, but it assumes that among the samples at least one is received with minimum delay. Furthermore it can only estimate the drift if the delay is small compared with $\rho R$. Both cannot be guarantied in a transputer network. 
[Christian 89] presents the following simple algorithm for node $i$ to approximate its difference with the clock value of $j$ and the accuracy of that approximation:
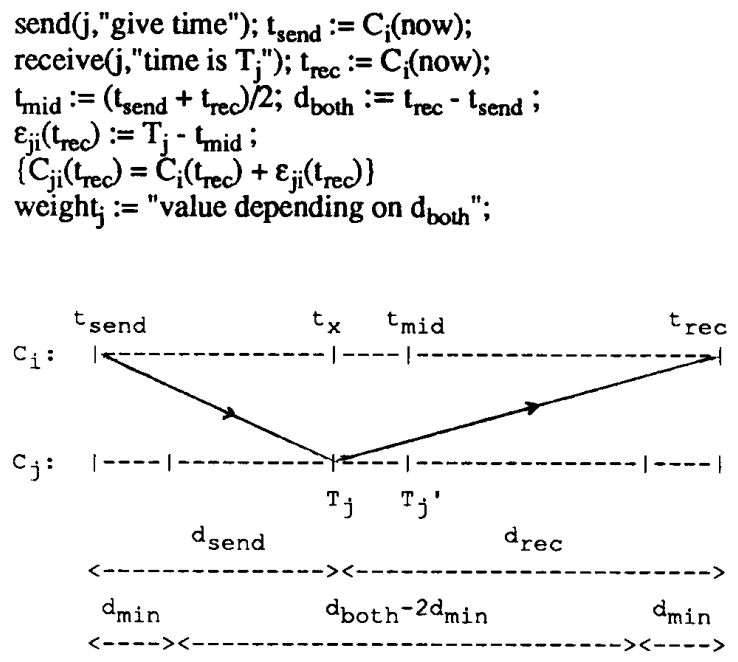

Node $i$ 's message is received by $j$ when $j$ 's clock reads $T_{j}$. As $i$ does not know this moment $t_{x}\left(t_{\text {send }}+d_{\text {send }}\right)$. it assumes reception at $t_{\text {mid. }}$. Its estimation $C_{j i}\left(t_{\text {mid }}\right)$ should have been $T_{j}^{\prime}$ instead of $T_{j}$. The error $T_{j}-T_{j}^{\prime}=t_{x}-t_{\text {mid }}=$ $\left(d_{\text {send }}-d_{\text {rec }}\right) / 2$, with a maximum of $\left(d_{\text {both }}-2 d_{\min }\right) / 2$, where $d_{\text {min }}$ is the minimum message delay. It increases with $\mathrm{d}_{\text {both }}$ and if confidence weight factors are to be used $\varepsilon_{\mathrm{ji}}\left(\mathrm{t}_{\mathrm{TeC}}\right)$ should get a lower weight $\mathrm{j}_{\mathrm{j}}$ the larger $\mathrm{d}_{\mathrm{both}}$ is.

Choice: In order not to accumulate unpredictable message delays the local approach is used: only the clocks of the direct neighbours in the transputer network are determined. Christians algorithm is used, with one sample per interval and with or without confidence weight factors. In the cold start one node acts as a master and its neighbours take its time and distribute it into the network.

\section{Estimation of the global time}

Possibilities: After the remote clock values $\mathrm{C}_{\mathrm{ji}}$ have been determined (let $U$ be the set of values including that of the local clock), the global time $G_{i}(t)$ has to be estimated by means of a convergence function. Possible convergence functions are:

$\mathrm{G}_{\mathrm{i}}(\mathrm{t})=\max (\mathrm{U}), \operatorname{mean}(\mathrm{U}), \min (\mathrm{U})$, or $\operatorname{median}(\mathrm{U})$.

If $U$ is a large set (which is not the case in a transputer network) the $\mathrm{m}$ smallest and largest values may be left out (resulting in U), to make the algorithm more fault tolerant. Examples are:

$\mathrm{G}_{\mathrm{i}}(\mathrm{t})=\operatorname{mean}\left(\mathrm{U}^{\prime}\right)$, or $\left(\max \left(U^{\prime}\right)+\min \left(U^{\prime}\right)\right) / 2$.
A node may also, if its trusts its own time, define a time window around its own clock value and only consider values in the window to belong to $U$.

Choice: With a local approach (neighbours only) the convergence function must be carefully chosen in order to avoid partitioning of the network. Simulations by [Rickert 88] have shown that mean(U) is a good choice for all phases. The confidence weight factors based on $d_{\text {both }}$ can be used to produce a weighted mean value.

\section{Adjustment of the local clock}

Possibilities: Once the estimated global time has been calculated the local clock must be adjusted to it. Two kinds of adjustments are possible: value (first order) and rate (second order) adjustment. Value adjustment may be done instantaneously, by adding the (possibly negative) difference 'at once' to the local clock value [Lamport 78, De Carlini 88] or gradually, by spreading the difference over the next resynchronization interval (in this case one could say that the clock rate is temporarily changed in such a way that the difference will have been added to the clock value by the end of the interval) [Christian 89 , Kopetz 87]. Both value adjustment methods may (or may not) be combined with rate adjustment, where the rate of the local clock is adjusted permanently in such a way that it may be expected that future differences will become smaller. A model which describes the above mentioned four Possible combinations is described below.

Suppose that at $t_{0}$ the local clock of node $i$ drifts away from the global time by $\rho_{i}\left(t_{0}\right)$ and that $\varepsilon_{i}\left(t_{n}\right)$ is the adjustment value for the next $\left(n^{\text {th }}\right)$ resynchronization interval $\left(t_{n}<t \leq t_{n+1}\right)$ of length $R$. The clock value at real time $t$ is defined as follows:

instantaneous adjustment only:

$$
C_{i}(t)=C_{i}\left(t_{n}\right)+\varepsilon_{i}\left(t_{n}\right)+\left(1+\rho_{i}\left(t_{0}\right)\right) \cdot\left(t-t_{n}\right)
$$

gradually adjustment only:

$$
\text { (2) } \quad C_{i}(t)=C_{i}\left(t_{n}\right)+\left(1+\varepsilon_{i}\left(t_{n}\right) / R+\rho_{i}\left(t_{0}\right)\right) \cdot\left(t-t_{n}\right)
$$

instantaneous plus rate adjustment (the instantaneous part is multiplied by $0 \leq \alpha \leq 1)$ :

$$
\text { (3) } \quad C_{i}(t)=C_{i}\left(t_{n}\right)+\alpha \cdot \varepsilon_{i}\left(t_{n}\right)+\left(1+\rho_{i}\left(t_{n}\right)\right) \cdot\left(t-t_{n}\right)
$$

gradually plus rate adjustment:

$$
\text { (4) } \quad C_{i}(t)=C_{i}\left(t_{n}\right)+\left(1+\alpha \cdot \varepsilon_{i}\left(t_{n}\right) / R+\rho_{i}\left(t_{n}\right)\right) \cdot\left(t-t_{n}\right)
$$

$$
\begin{aligned}
& \text { In (3) and (4) } \rho_{i}\left(t_{n}\right) \text { is recursively defined by } \\
& \qquad \rho_{i}\left(t_{n}\right)=\rho_{i}\left(t_{n-1}\right)+\beta \cdot \varepsilon_{i}\left(t_{n}\right) / R
\end{aligned}
$$

with $0 \leq \beta \leq 1$.

The first two are special cases of the last two with $\alpha=1$ and $\beta=0$. The last two lead to the same value for $C\left(t_{n+1}\right)$ 
at the end of resynchronization interval. Only within the interval the local clock values differ.

In the last two cases the differences of the past are filtered by a second order filter [Meijerink 89]. The size of $\alpha$ and $\beta$ determines the settling speed and the damping of the filter respectively.

Choice: In the cold and warm start instantaneous adjustments are used to bring the clocks quickly together. In the warm start not only the value but also the rate of the "fresh" node is adjusted before it transfers to steady state. In order to avoid negative adjustments in the steady state, gradually combined with rate adjustment is used. As a result resynchronization intervals may grow larger because the drifts of the clocks will get closer together.

\section{Simulation}

\section{The simulated items with their parameter values}

Clocks.

Each node $\mathrm{i}$ is assumed to have a local clock $\mathrm{C}_{\mathrm{i}}(\mathrm{t})$ with a drift $\rho_{i}(t)$. To study the drift convergence capability of the algorithm the drift is initiallized with a value uniformly chosen from the interval:

$\left[-10^{-4}, \ldots, 10^{-4}\right]$. The clocks are initially assigned a value such that $\Delta=10^{-4}$. The simulated time is divided into units of length step. If step time has passed, rate*step is added to the clock, where rate is $1+\alpha \cdot \varepsilon_{i}\left(t_{n}\right) / R+\rho_{i}\left(t_{n}\right)$, the adjusted clock rate for the current resynchronization interval (see equation 4 above). Intuitively the smaller the size of step, the more accurate the results of the simulation. Some experimental runs proved that this effect is negligible. To speed up the simulation a bit the value of step was set equal to $R / 5$, where $R$ is the length of the resynchronization interval. The size of $R$ has arbitrary been chosen: $1,10,25$ and 50 seconds.

Node i starts the resynchronization algorithm if the time on its clock exceeds its next resynchronization moment $\mathrm{t}_{\mathrm{s}}$. It then reads its neighbour clock values immediately.

Messages.

Instead of sending and receiving real messages, a remote clock $C_{j}$ is read by node $i$ at the moment of resynchronization $t_{s}$. To simulate the error due to non predictable message delays two delays $d_{\text {send }}$ and $d_{\text {rec }}$ are taken from an Erlang distribution and the error $T_{j}-T_{j}=$ $\left(d_{\text {send }}-d_{\text {rec }}\right) / 2$ is added to $C_{j}\left(t_{s}\right)$.

The Erlang probability density function is defined by

$$
f(x)=\left(\lambda^{n} x^{n-1} e^{-\lambda x}\right) /(n-1) ! \quad(0 \leq x)
$$

For $n=2$ the mean message delay is $2 / \lambda$ and the standard deviation is $\sqrt{ }(2 / \lambda)$. The mean delay of both messages together is $4 / \lambda$. Simulations are performed with $4 \lambda=10^{-3}$ and $10^{-4} \mathrm{sec}$, corresponding with a mean two way message delay $d_{\text {both }}$ of $1 \mathrm{~ms}$ and $0.1 \mathrm{~ms}$ respectively.

Confidence.

Let $F(x)$ be the probability function of the Erlang distribution. A good function to calculate a confidence weight is $1-F(x)$. Note that $x=\lambda^{*} d_{\text {both }} / 8$, corresponding with $x=0.5$ for a mean delay $d_{\text {both }}$ of $4 \lambda$.

In the simulation weight $t_{j}=1-F(x)$ is approximated by

$$
\begin{array}{ll}
1 & \text { if } 0 \leq x \leq 0.45 \\
-0.85 x+1.3 & \text { if } 0.45 \leq x \leq 1.15 \\
0 & \text { if } 1.15 \leq x
\end{array}
$$

During the first 40 resynchronizations no use is made of a confidence weight factor. To get an idea of the influence of using confidence, all simulations were carried out with and without the use of weight factors.

\section{Local clock adjustment.}

The values of $\alpha$ and $\beta$ determine the settling speed and damping of the second order adjustment filter. Simulations by [Meijerink 89] for an ethernet gave the best results with 0.2 and 0.022 respectively so we started simulating with those values. Experiments with other values are planned.

The first nine resynchronization intervals take different values to bring the clocks quickly together:

$\mathbf{n}=1 . .3: \alpha=1.0 / \mathbf{n} ; \beta=0.3 / \mathbf{n}$
$\mathbf{n}=4 . .9: \alpha=0.3 ; \quad \beta=0.053$
$\mathbf{n}=10 . .2 \alpha=0.2 ; \quad \beta=0.022$

\section{Topologies.}

Two homogeneous topologies have been simulated: a 10 $x \mathbf{1 0}$ torus and a ring of 20 nodes. The torus is chosen to investigate how the algorithm acts in a network where each node has a maximum number of neighbours (=4). A ring of 20 nodes is chosen since the diameter of the ring is equal to the diameter of the $10 \times 10$ torus, namely 10 . The effect of the smaller number of neighbours $(=2)$ can be compared with the results of the torus.

\section{Programming environment.}

The simulation is programmed in Turbo Pascal 5.0. and is executed on an IBM-compatible PC. The Erlang distributed message delays are generated by a program written in Turbo C 2.0 using algorithms described in [MacDougall 87]. 


\section{Results of the simulations}

The results of the 10 by 10 torus and 20 nodes ring simulation are presented in figures 1 to 6 below. They show the influence of the non-deterministic message delay on the convergence of the algorithm.

Parameters for the simulations are: $\mathbf{R}(1,10,25,50 \mathrm{sec})$; $\lambda\left(4.10^{3}, 4.10^{4} \mathrm{sec}^{-1}:\right.$ mean $\mathrm{d}_{\text {both }}=1$ resp. $\left.0.1 \mathrm{msec}\right)$ and the (yes or no) use of confidence weight factors. In the figures below codes $3,3 \mathrm{c}, 4$ and $4 \mathrm{c}$ refer to a simulation result with $\lambda=4.10^{3}$, without resp. with confidence and $\lambda=4.10^{4}$, without resp. with confidence. Each simulation consists of 500 resynchronizations and is carried out twice for each set of parameters. Differences in both simulations with the same parameter values were in the order of $10 \%$.

Two values were calculated during the simulations: the maximum clock difference $\Delta_{\text {clock }}$ and maximum rate difference $\Delta_{\text {rate. }}$ Examples of their behaviour as a function of the number of resynchronizations are presented in figures 1 and 2 . The mean value over the last 100 resynchronizations of $\Delta_{\text {clock }}$ and $\Delta_{\text {rate }}$ are presented in figures 3 to 6.

\section{Discussion.}

As expected quick synchronization is obtained for short resynchronization intervals: figure 1 shows that for $R=1$ all curves remain below $\Delta=0.1 \mathrm{~d}=1 \mathrm{msec}$ after about 25 resynchronizations.

Figures 3 and 4 show that $\Delta_{\text {clock }}$ is proportional to $\mathrm{R}$. The influence of the message delay on the mean of $\Delta_{\text {clock }}$ over the last 100 resynchronizations is less than the influence of confidence. The algorithm behaves better without the use of confidence: With confidence $R=25$ seems to be the limit to keep clock differences below $0.1 \mathrm{~d}$ msec, whereas $\mathrm{R}=50$ is possible without confidence. Since there is only a small number of remote clocks, too many of them could easily get a zero confidence weight factor, resulting in poor adjustment of the local clock. Above that the filter indirectly associates a kind of confidence with the calculated global time too (a small $\alpha$ means that a difference is only partially corrected). An optimal relation between the confidence function and the values for $\alpha$ and $\beta$ has not yet been found.

Figure 4 shows that the algorithm behaves a bit worse on the ring than on the torus, probably because there are less neighbours to correct the local time.
Figures 5 and 6 show that $\Delta_{\text {rate }}$ does not differ much for torus and ring. For $R>10$ the mean of $\Delta_{\text {rate }}$ over the last 100 resynchronizations is between $10^{-5}$ and $10^{-6}$ for both $\lambda$ 's, with or without confidence. For small $R$ the drift seems to be corrected too frequently. Possibly other values for $\alpha$ and $\beta$ are needed in those cases.

Figures 1 and 2 show that for small delays $\Delta_{\text {clock }}$ and $\Delta_{\text {rate }}$ have an oscillating behaviour. It is expected that it is caused by the second order filter, but a suitable explanation has not yet been found.

\section{Conclusion}

Simulations on the ring and the torus (both with diameter $d=10$ ) show that the proposed algorithm realizes the two requirements stated in the beginning: (1) to bring and keep $\Delta_{\text {clock }}$ below $\Delta=0.1 \mathrm{~d}=1 \mathrm{msec}$ and (2) to decrease $\Delta_{\text {rate }}$

\section{References}

[Christian 89] Christian, F., "Probalistic clock synchronization", Distributed Computing, 1989, Vol. 3, No. 3, Pp. 146-158.

[De Carlini 88] De Carlini, U. and U. Villano, "A Simple Algorithm for Clock Synchronization in Transputer Network", Software Practice and Experience, 1988, Vol. 18, No.4, April, pp. 331-347.

[Kopetz 87] Kopetz, H. and W. Ochsenreiter, "Clock Synchronization in Distributed Real-Time Systems", IEEE Transactions on computers, 1987, Vol. C-36, No. 8, August, pp. 933-940.

[Lamport 78] Lamport, L., "Time, Clocks and the Ordering of Events in a Distributed System", Communications of the ACM, 1978, Vol 21, No. 7, July, pp. 558-565.

[Lamport 85] Lamport, L. and P.M. Melliar-Smith "Synchronizing Clocks in the Presence of Faults", Joumal of the ACM 1985, Vol. 32, No. 4, January, pp. 52-78.

[MacDougall 87] MacDougall, M.H., Simulating Computer Systems: Techniques and Tools, The MIT Press, 1987, MIT Press Series in Computer Systems.

[Meijerink 89] Meijerink, G.J., Clock Synchronization in a distributed Real-time Systems, MSc Thesis, University of Twente, The Netherlands, 1989.

[Ricken 88] Rickert, N.W., "Non byzantine clock synchronization - a programming experiment", Operating Systems synchronization - a programming experiment", Opper
Review, 1988, Vol. 22, No. 1, January, pp. 73-78.

[Srikanth 87] Srikanth, T.K. and S. Toueg, "Optimal Clock Synchronization", Joumal of the ACM, 1987, Vol. 34, No. 3, July, pp. 625-645.

[Thambidurai 89] Thambidurai, P., A.M. Finn, R.M. Kieckhafer and C.J. Walter, "Clock Synchronization in MAFT", The nineteenth intemational symposium on fault tolerant computing, 1989, pp. 142internati 


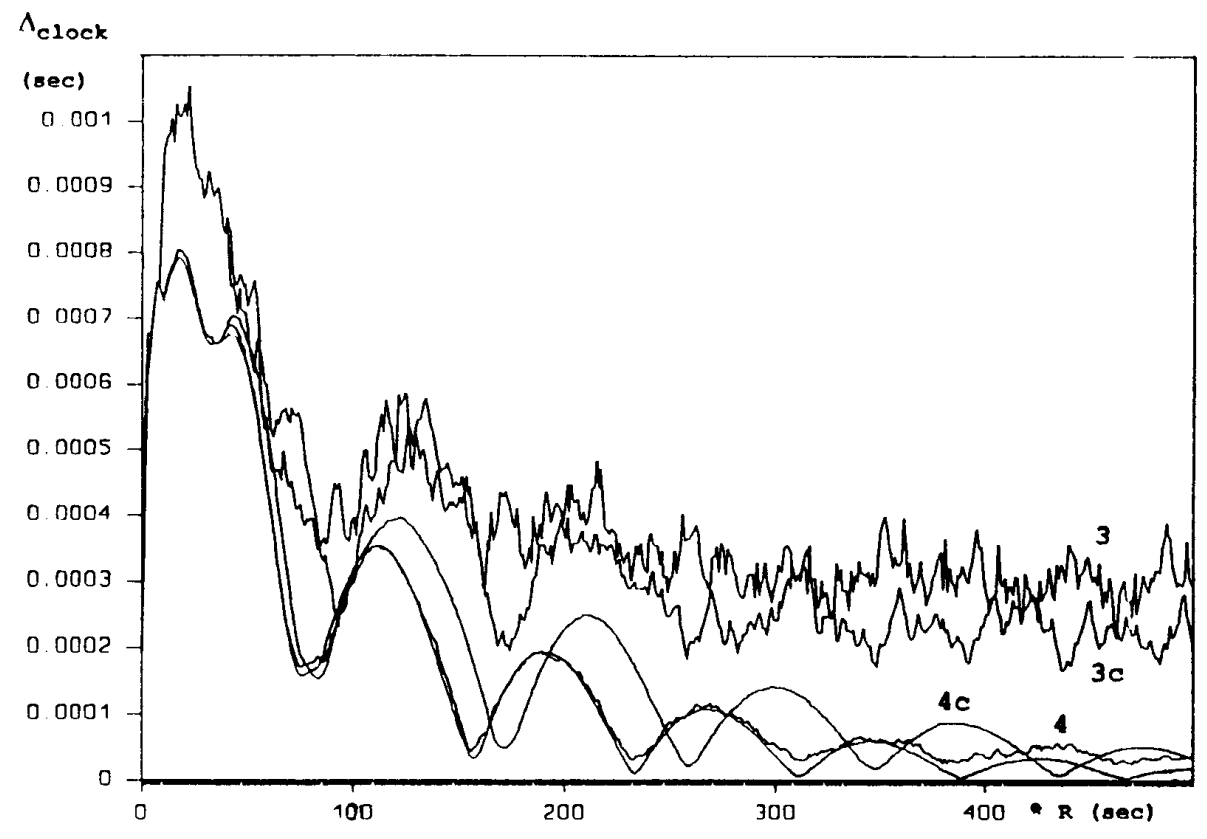

fig 1. $\Delta_{\text {clock }}$ for $10^{*} 10$ TORUS, $R=1$

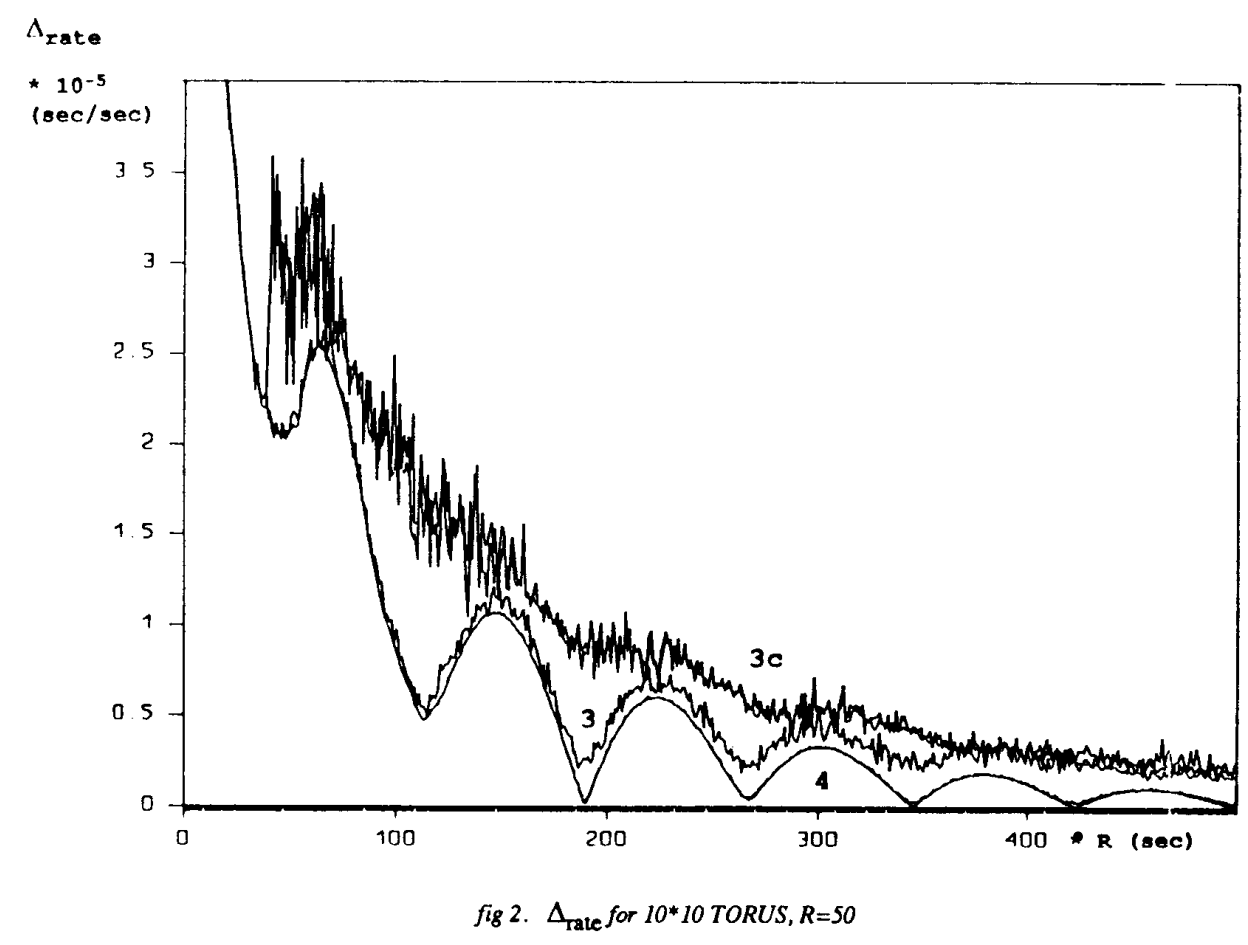




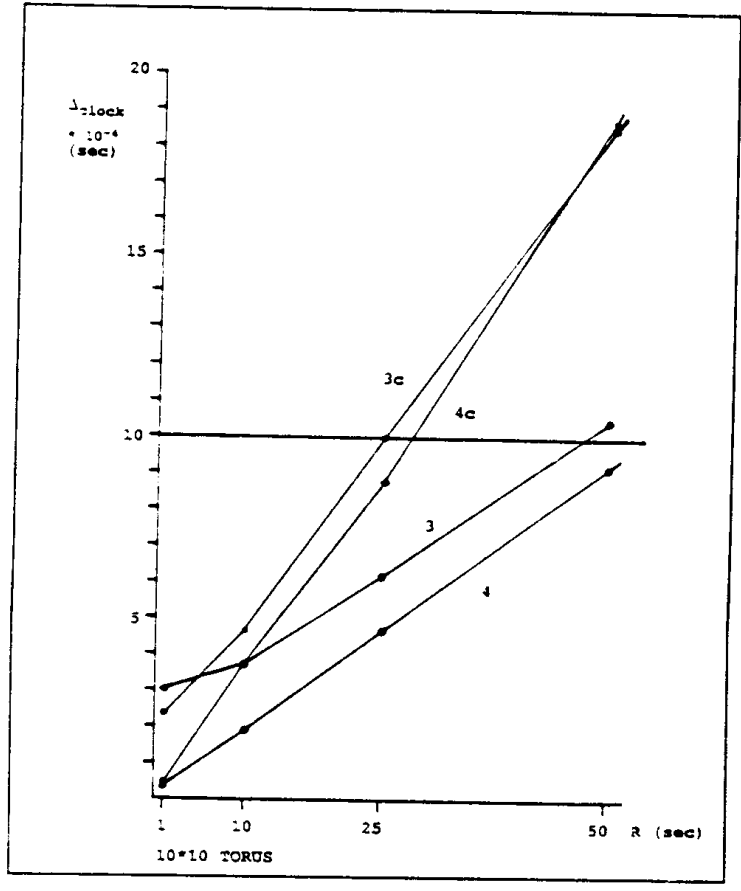

fig 3. mean $\Delta_{\text {clock }}$ for 10*10 TORUS

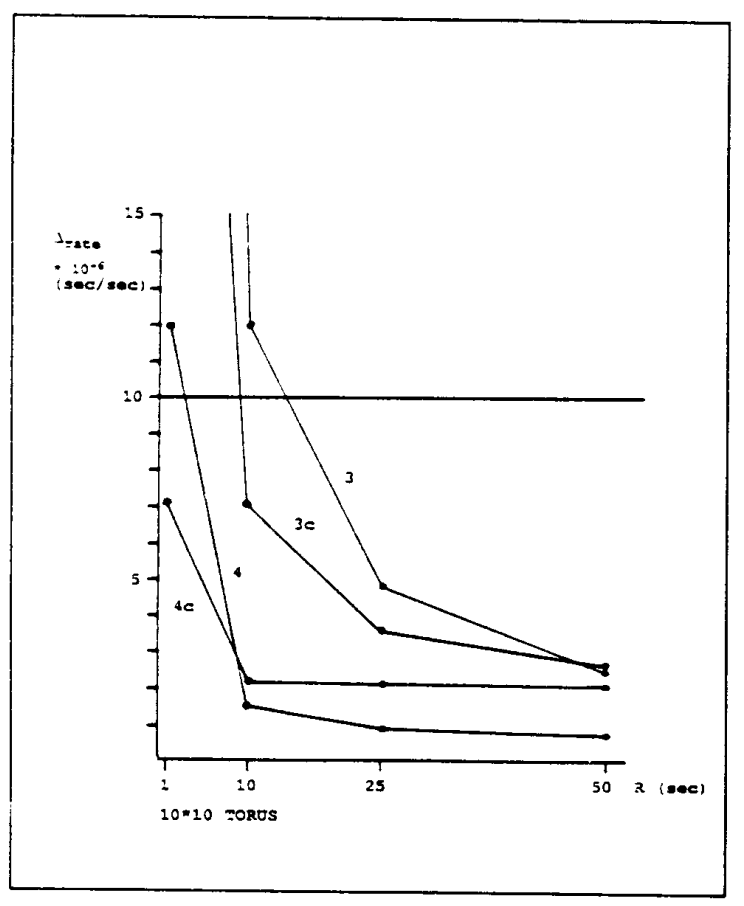

fig 5. mean $\Delta_{\text {rate }}$ for 10*10 TORUS

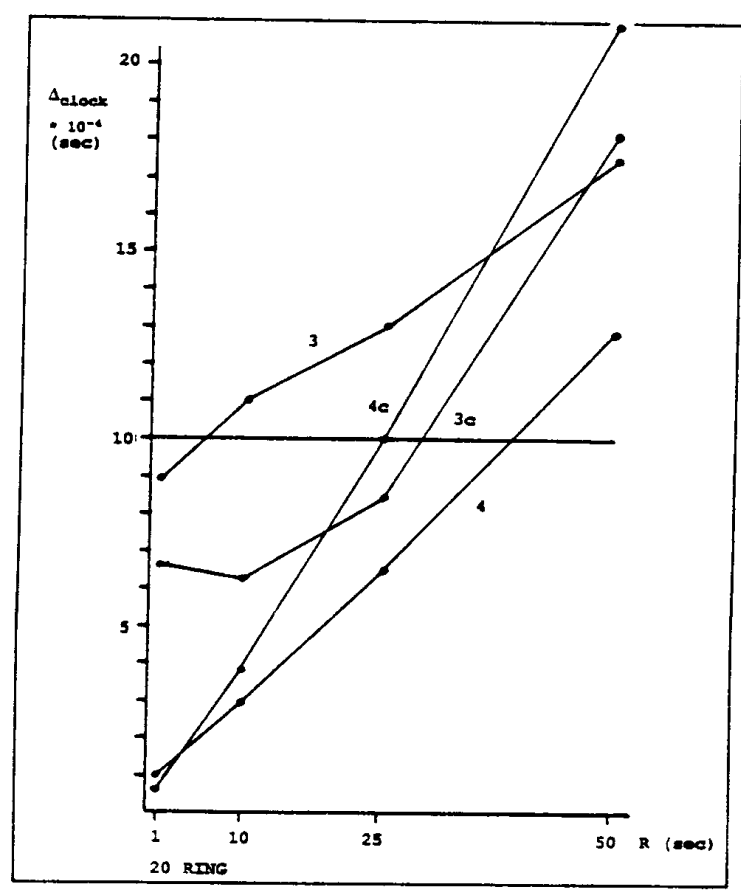

fig 4. mean $\Delta_{\text {clock }}$ for 20-RING

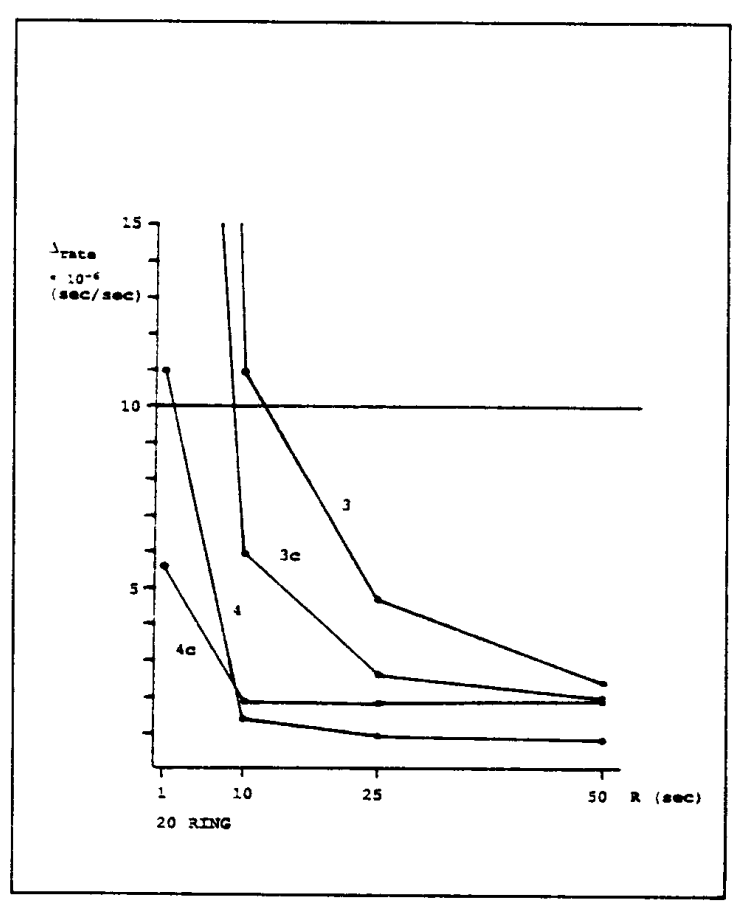

fig 6. mean $\Delta_{\text {rate }}$ for 20-RING 